IASSNS-HEP-94/68

\title{
Iterated Moiré Maps and Braiding of Chiral Polymer Crystals
}

\author{
Randall D. Kamien ${ }^{1}$ \\ School of Natural Sciences, Institute for Advanced Study, Princeton, NJ 08540 \\ and \\ David R. Nelson \\ Lyman Laboratory of Physics, Harvard University, Cambridge, MA 02138
}

\begin{abstract}
In the hexagonal columnar phase of chiral polymers a bias towards cholesteric twist competes with braiding along an average direction. When the chirality is strong, screw dislocations proliferate, leading to either a tilt grain boundary phase or a new "moiré state" with twisted bond order. Polymer trajectories in the plane perpendicular to their average direction are described by iterated moiré maps of remarkable complexity.
\end{abstract}

9 November 1994

1 email: kamien@guinness.ias.edu 
A notable feature of biological materials is the profusion of long polymer molecules with a definite handedness. DNA, polypeptides (such as poly- $\gamma$-benzyl-glutamate) and polysaccharides (such as xanthan) can all be synthesized with a preferred chirality. Long polymers in dense solution often crystallize into a hexagonal columnar phase. When the polymers are chiral this close packing into a triangular lattice competes with the tendency for the polymers to twist macroscopically [1] as in cholesteric liquid crystals. Similar to the twist grain boundary phase of chiral smectics [2], macroscopic chirality can proliferate when screw dislocations enter the crystal. Like flux lines in a type II superconductor, dislocations only appear provided the free energy reduction from the chiral couplings exceeds the dislocation core energy. If the chirality is weak, a defect free hexagonal columnar phase persists, as in the Meissner phase of superconductors.

In this letter we explore the effect of chirality on the hexagonal columnar phases [3] of long polymers in detail. We neglect for simplicity heterogeneity along the polymer backbones and work with a two component displacement field perpendicular to the local polymer direction. The usual chiral term relevant for cholesteric liquid crystals produces the polymer tilt grain boundary phase, similar to the smectic- $A^{*}$ phase [2]. We find, as well, an additional term in the free energy which favors the rotation of the bond order along the average polymer direction. This term leads to braided polymers with twisting describable by a sequence of moiré patterns.

Using continuum elastic theory we estimate the critical values of the chiral couplings above which screw dislocations enter. On a more microscopic level, we propose a set of lock-in moiré textures which should be especially low in elastic energy. These states are entangled and self-similar, and lead to tortuous polymer paths of remarkable complexity, reminiscent of chaotic dynamical systems.

When polymer nematics crystallize the areal polymer density in a plane perpendicular to the average direction may be approximated as

$$
\rho \approx \rho_{0}+\sum_{\alpha} \rho_{\alpha}(\vec{r}) \exp \left\{-i \vec{G}_{\alpha} \cdot \vec{r}\right\}
$$


where the $\left\{\vec{G}_{\alpha}\right\}$ are the six smallest reciprocal lattice vectors of a triangular lattice. We take the average polymer direction here and throughout to be $\hat{z}$, with $\vec{G}_{\alpha} \cdot \hat{z}=0$. Each plane wave is modulated by a spatially varying magnitude and phase $\rho_{\alpha}(\vec{r})=$ $\left|\rho_{\alpha}(\vec{r})\right| \exp \left\{i \vec{G}_{\alpha} \cdot \vec{u}(\vec{r})\right\}$, where $\vec{u}$ is a two-dimensional displacement field. In addition, we have a nematic order parameter, $\hat{n} \approx \hat{z}+\delta \vec{n}$ and, in the case of a triangular lattice of polymers, a bond field $\theta_{6}$ which measures the bond-orientational order in the $x y$-plane, modulo $2 \pi / 6$. Under a global rotation about the $x$-axis or $y$-axis by an angle $\theta_{x}$ or $\theta_{y}$ respectively, $\hat{n} \rightarrow \hat{n}+\vec{\theta} \times \hat{n} \approx \hat{z}+\theta_{y} \hat{x}-\theta_{x} \hat{y}$, i.e. $\delta \vec{n} \rightarrow \delta \vec{n}+\theta_{y} \hat{x}-\theta_{x} \hat{y}$. Similarly under a global rotation about the $z$-axis by $\theta_{z}, \theta_{6} \rightarrow \theta_{6}+\theta_{z}$. Under such rotations, $\vec{r} \rightarrow \vec{r}-\vec{\theta} \times \vec{r}$, leading to a position dependent change in the phase of $\rho_{\alpha}(\vec{r})$. To insure rotational invariance, derivatives of $\rho_{\alpha}(\vec{r})$ must be accompanied by the fields $\theta_{6}$ and $\delta \vec{n}[4]$ and we are led to the Landau free energy

$$
\begin{aligned}
\mathcal{F}_{\mathrm{d}}= & \sum_{\alpha}\left\{\frac{A}{2}\left|\vec{G}_{\alpha} \cdot\left[\nabla_{\perp} \rho_{\alpha}-i \theta_{6}\left(\vec{G}_{\alpha} \times \hat{z}\right) \rho_{\alpha}\right]\right|^{2}+\frac{B}{2}\left|\vec{G}_{\alpha} \times\left[\nabla_{\perp} \rho_{\alpha}-i \theta_{6}\left(\vec{G}_{\alpha} \times \hat{z}\right) \rho_{\alpha}\right]\right|^{2}\right. \\
& \left.+\frac{C}{2}\left|\partial_{z} \rho_{\alpha}-i\left(\vec{G}_{\alpha} \cdot \delta \vec{n}\right) \rho_{\alpha}\right|^{2}+\frac{b}{2}\left|\rho_{\alpha}\right|^{2}\right\}+c \sum_{\alpha \beta \gamma}^{\vec{G}_{\alpha}+\vec{G}_{\beta}+\vec{G}_{\gamma}=\overrightarrow{0}} \rho_{\alpha} \rho_{\beta} \rho_{\gamma}+\mathcal{O}\left(\rho_{\alpha}^{4}\right)
\end{aligned}
$$

Crystalline order arises for $b$ sufficiently negative so that $\left\langle\rho_{\alpha}\right\rangle \neq 0$. Due to the third order term, this transition will, in general, be first order.

To this free energy we add two more pieces. It is convenient, but not essential, to imagine that the hexagonal columnar density waves arise from a phase with local nematic order as well as six-fold bond order perpendicular to the director axis. This " $\mathrm{N}+6$ " phase has been studied by Toner [5]. Although the present experimental evidence for $\mathrm{N}+6$ order in polymer nematics is sketchy, such phases seem highly likely in columnar systems, in analogy with the hexatic order expected for vortex lines in high temperature superconductors [6]. In the absence of chirality we have the usual elastic energies of the nematic field and the bond angle field,

$$
\mathcal{F}_{\mathrm{o}}=\frac{1}{2}\left[K_{1}\left(\nabla_{\perp} \cdot \delta \vec{n}\right)^{2}+K_{2}\left(\nabla_{\perp} \times \delta \vec{n}\right)^{2}+K_{3}\left(\partial_{z} \delta \vec{n}\right)^{2}+K_{A}^{\|}\left(\partial_{z} \theta_{6}\right)^{2}+K_{A}^{\perp}\left(\nabla_{\perp} \theta_{6}\right)^{2}\right]
$$


where the $\left\{K_{i}\right\}$ are Frank constants, and $K_{A}^{\|}$and $K_{A}^{\perp}$ are hexatic stiffnesses parallel and perpendicular to $\hat{z}$. For long chain polymers $K_{1} \gg K_{2}, K_{3}[7]$.

Finally, we add those chiral terms which respect the nematic symmetry. If there is no preferred direction along the polymers then the free energy must be invariant under $\hat{n} \rightarrow-\hat{n}$. Additionally, since changes in $\theta_{6}$ are measured with respect to the $\hat{n}$ axis, under nematic inversion, $\theta_{6} \rightarrow-\theta_{6}$, and the vector $\vec{v} \equiv \nabla \theta_{6}$ changes sign. Under spatial inversion $\hat{n} \rightarrow-\hat{n}, \theta_{6} \rightarrow-\theta_{6}$ and hence $\vec{v} \rightarrow \vec{v}$. The chiral free energy density $\mathcal{F}^{*}$ contains two distinct terms invariant under nematic inversion but which change sign under parity [8],

$$
\mathcal{F}^{*}=-K_{2} q_{0} \hat{n} \cdot(\nabla \times \hat{n})-K_{A}^{\|} \tilde{q}_{0} \vec{v} \cdot \hat{n} \approx-\gamma \nabla_{\perp} \times \delta \vec{n}-\gamma^{\prime} \partial_{z} \theta_{6}
$$

with $\gamma=K_{2} q_{0}$ and $\gamma^{\prime}=K_{A}^{\|} \tilde{q}_{0}$. The first term often leads to cholesteric twist with period $2 \pi / q_{0}$ along a line perpendicular to the plane in which the nematic director lies. The second induces twist into the bond-order parameter $\theta_{6}$ with period $2 \pi / \tilde{q}_{0}$ along $\hat{z}$. The consequences of this additional chiral coupling is the main subject of this paper. The total free energy is $F=\int d^{3} r\left[\mathcal{F}_{\mathrm{d}}+\mathcal{F}_{\mathrm{o}}+\mathcal{F}^{*}\right]$. Note the close similarity between (2)-(4) and the Ginsburg-Landau theory of a superconductor in a magnetic field. The fields $\delta \vec{n}$ and $\theta_{6}$ are "gauge fields" minimally coupled to the complex order parameters $\left\{\rho_{\alpha}(\vec{r})\right\}$ by the constraint of rotational invariance. There are two distinct "magnetic" fields $\gamma$ and $\gamma^{\prime}$.

Provided $b \ll 0$ we can set $\rho_{\alpha}=\left|\rho_{0}\right| \exp \left\{i \vec{G}_{\alpha} \cdot \vec{u}(\vec{r})\right\}$ and minimize $F$ to find $\delta n_{i}=\partial_{z} u_{i}$ and $\theta_{6}=\frac{1}{2} \epsilon_{i j} \partial_{i} u_{j}$. The resulting elastic free energy is now

$$
F=\int d^{3} r\left\{\mu u_{i j}^{2}+\frac{\lambda}{2} u_{i i}^{2}+K_{3}\left(\partial_{z}^{2} u_{i}\right)^{2}-\gamma \nabla_{\perp} \times \delta \vec{n}-\gamma^{\prime} \partial_{z} \theta_{6}\right\}
$$

where $u_{i j}=\frac{1}{2}\left(\partial_{i} u_{j}+\partial_{j} u_{i}\right), \mu=\frac{3}{4}|\vec{G}|^{4}\left|\rho_{0}\right|^{2}(A+B)$ and $\lambda=\frac{3}{4}|\vec{G}|^{4}\left|\rho_{0}\right|^{2}(A-B)$. In the columnar crystal the two chiral terms are the same if $\partial_{z} \partial_{i}=\partial_{i} \partial_{z}$. However, in the presence of dislocations derivatives do not commute, $\nabla_{\perp} \times \delta \vec{n}=\epsilon_{i j} \partial_{i} \partial_{z} u_{j} \neq \partial_{z} \epsilon_{i j} \partial_{i} u_{j}=\partial_{z} \theta_{6}$. Here, and throughout, $i, j \ldots$ refer to indices in the $x y$-plane. Burgers vectors of dislocations in a hexagonal columnar phase lie in the $x y$-plane, and there are three generic types [3]: a screw 
dislocation, an edge dislocation with tangent along $\hat{z}$, and an edge dislocation lying in the $x y$-plane. The latter defect requires aligned polymer ends which we neglect in this paper. The remaining dislocations must lie in a plane spanned by their Burgers vector $\vec{b}$ and $\hat{z}$, which amounts to choosing dislocation complexions with $\alpha_{x y}=\alpha_{y x}$, where the dislocation density tensor $\alpha_{\gamma i}$ is the density of dislocations with tangents along the $\gamma$-direction with Burgers vectors pointing in the $i$ direction [6].

Proceeding as in $[9,6]$ we introduce a new field $w_{\gamma i} \equiv \partial_{\gamma} u_{i}$ away from any dislocations. The non-commutivity of the derivatives of $\vec{u}$ is represented by the dislocation density, $\epsilon_{\mu \nu \gamma} \partial_{\nu} w_{\gamma i}=-\alpha_{\mu i}$. One can solve for $w_{\gamma i}$ in terms of the dislocation density $\alpha_{\gamma i}$ and find the equilibrium displacement field in the presence of crystal defects [10]. In terms of the nematic and bond order field non-commutivity of derivatives means $2 \partial_{z} \theta_{6}-\nabla_{\perp} \times \delta \vec{n}=-\operatorname{Tr}[\alpha]$. The energy per unit length of a screw dislocation is finite, while that of an edge dislocation lying along the $\hat{z}$ direction diverges logarithmically with system size [11]. The screw dislocation energy depends on the dimensionless parameter $\delta=\left(K_{3} \xi_{\perp}^{2} / \mu \xi_{z}^{4}\right)^{1 / 4}$, where $\xi_{\perp}$ and $\xi_{z}$ are short distance cutoffs in the perpendicular and parallel directions, respectively. We estimate $\delta \gg 1$ for polymer crystals which is the condition that the response to chirality be similar to that of Type II as opposed to Type I superconductors [2]. In this limit the free energy per unit length of a screw dislocation is $f_{\mathrm{s}}=\left(\mu^{3 / 4} K_{3}^{1 / 4} b^{2}\right) /\left(2 \pi \sqrt{2 \xi_{\perp}}\right)$, where $b$ is the length of the Burgers vector.

If the chirality is strong and $\gamma \gg \gamma^{\prime}$ we expect the polymer analogue of the RennLubensky twist-grain-boundary state [2]. Each of these tilt grain boundaries (TGB) is composed of a parallel array of screw dislocations lying, say, in the $x z$-plane, pointing along the $x$-axis and uniformly spaced along $\hat{z}$ with spacing $d$. As illustrated in Figure 1 this dislocation texture causes a discrete rotation $\phi=\tan ^{-1}(b / d)$ in the average polymer direction. The spatial integral of $\nabla_{\perp} \times \delta \vec{n}$ is non-zero, while the integral of $\partial_{z} \theta_{6}$ vanishes. The TGB state appears when the chiral coupling $\gamma$ exceeds the critical value $\gamma_{c}=f_{\text {screw }} / b$. The diffraction signature is similar to that of the smectic- $A^{*}$ phase: if the pitch axis is $\hat{y}$ so that 
the polymers lie, on average, in the $x z$-plane, the structure function would consist of two Bragg circles with radius $2 \pi / a$ lying in a $q_{x} q_{z}$-plane and centered on $\vec{q}=(0, \pm 2 \pi / \sqrt{3} a, 0)$ where $a_{0}$ is the lattice constant and two Bragg spots at $q_{y}= \pm 4 \pi / \sqrt{3} a_{0}$. These features should have widths $\sim 2 \pi / d^{\prime}$ along $\hat{q}_{y}$ where $d^{\prime}$ is the spacing between tilt grain boundaries. If the rotation angle between successive crystalline regions were a rational fraction of $2 \pi$, the Bragg circles would break up into a sequence of Bragg spots spaced around the circle. Although a precise determination of $d$ and $d^{\prime}$ would require more detailed energetic calculations, we estimate, following [2], that $d \approx d^{\prime} \approx \sqrt{a_{0} / q_{0}}$.

The second chiral coupling $\gamma^{\prime}$ has no analogue in chiral smectics. To find a configuration of screw dislocations which exploits this form of chirality we search for a dislocation texture which produces a displacement depending only on $z$. The only texture which does not produce divergent elastic energy is one in which the only non-zero components of $\alpha_{\gamma i}$ are $\alpha_{x x}=\alpha_{y y}[6,10]$. A honeycomb array of screw dislocations, on average, produces this dislocation texture. We now find that for a single such grain boundary $\nabla_{\perp} \times \delta \vec{n}$ vanishes far from the boundary while $\partial_{z} \theta_{6}$ does not. This sort of grain boundary thus causes a net twist of the hexatic order parameter $\theta_{6}$ while imposing no net $\nabla_{\perp} \times \delta \vec{n}$. When $\gamma^{\prime} \gg \gamma$ and the chirality is large, screw dislocations penetrate for $\gamma^{\prime}>\gamma_{c}^{\prime}=2 f_{\mathrm{s}} / b$. By choosing the rotation angle across the honeycomb dislocation network to produce a high density of coincidence lattice sites [12], we produce especially low strain energies across the boundary. The superposition of triangular polymer lattices below and above the boundary forms a moiré pattern. The superposition of many such "moiré sheets" along the $z$-axis braids the polymers with deep minima in the energy at certain lock-in angles. Figure 1 illustrates the mapping of polymers across the moiré plane, for a rotation angle $\phi_{2} \approx 13.2^{\circ}$ with a particularly dense set of coincidence lattice sites. Polymers in the lower half-space (circles) must be connected to the closest available polymer in the upper half-space (crosses) to minimize bending energy. Note that the map has a discrete translational symmetry, in the sense that any coincidence site could be a center of rotation. Especially simple 
moiré maps arise for rotation angles $\phi_{n}=2 \tan ^{-1}[\sqrt{3} / 3(2 n+1)], n=1,2, \ldots$. It can be shown that all such angles are irrational fractions of $2 \pi$ [10] so that the structure never repeats upon iteration. Around each coincidence point there are $n$ concentric rings of helical polymers The lattice of coincidence points is also a triangular lattice, but with a spacing $a_{n}=a_{0} \sqrt{1+3(2 n+1)^{2}} / 2$, where $a_{0}$ is the original lattice spacing. The geometrical origin of such energetically preferred lock-in angles has no analogue in chiral smectics. The exact choice of lock-in angles and spacing between moiré planes must again be settled by detailed energetic calculations.

Upon two iterations of the moiré map separating three regions of polymer crystal, the first coincidence lattice is rotated with respect to the second coincidence lattice by precisely the angle of rotation $\phi_{n}$. Thus the composite coincidence lattice is the "coincidence lattice of coincidence lattices", with lattice constant $a_{n}^{2} / a_{0}$. Moiré maps iterated $p$ times lead to triangular composite coincidence lattices with spacing $a_{n}\left(a_{n} / a_{0}\right)^{p-1}$, i.e. to ever sparser lattices of fixed points with intricate fractal structure in between them. Figure 2 shows the projected polymer paths for $p=3$ and $n=1$. In contrast to the TGB state polymers for $p \gg 1$ are highly entangled and wander far from straight line trajectories. The polymer configurations and the dislocations leading to them are shown in Figure 3 for $n=1$. Near the moiré planes both $\theta_{6}$ and $\nabla_{\perp} \times \delta \vec{n}$ are nonzero. The center polymer is a fixed point of all the maps. Any such fixed polymer has a halo of others twisting around it. In this special tube, the nematic order parameter takes on the texture of a double twist cylinder as found in the low-chirality limit of blue phases [13]. The moiré state takes advantage of both double twist energies and the new chiral coupling $\gamma^{\prime}$. Closely related states are possible in the chiral $\mathrm{N}+6$ phases. Textures of vortex lines in Type II superconductors, similar to Figure 3, may arise when the Abrikosov flux lattice is subjected to a strong supercurrent parallel to the field direction [10].

In the inset to Figure 2 we show a random selection of polymer paths projected onto the $x y$-plane representing 99 iterations of the moiré map. Though these polymers are 
clearly influenced by the exceptional center point which is a fixed point of all 99 mappings, there are still paths of great complexity such that polymers wander far from their original positions. In a defect-free hexagonal columnar phase the most intense Bragg spots appear in the $q_{z}=0$ plane at the vertices of a hexagon with radius $G=4 \pi / \sqrt{3} a_{0}$. The braided crystal described by the iterated moiré map should exhibit a Bragg ring at $\left|\vec{q}_{\perp}\right|=G$ in the $q_{z}=0$ plane. The ring should be very sharp along $\vec{q}_{\perp}$, and of width $\sim 2 \pi / d^{\prime}$ along $\hat{q}_{z}$, where $d^{\prime}$ is the spacing between moiré planes. We estimate that $d^{\prime} \approx a_{n} \approx \sqrt{a_{0} / \tilde{q}_{0}}$. As in the Renn-Lubensky state [2], rational moiré rotations with angle $\phi=2 \pi s / t$, where $s$ and $t$ are relatively prime integers, would cause the Bragg rings to break up into $t$ spots spaced out around the ring, with additional structure along $\hat{q}_{z}$.

The phase diagram in Figure 1 summarizes our conclusions. The hexagonal columnar phase is like the Meissner phase of Type II superconductors. The two chiral couplings $\gamma$ and $\gamma^{\prime}$ cause screw dislocations to penetrate the crystal above critical strengths $\gamma_{c}$ and $\gamma_{c}^{\prime}$, in much the same way as vortices enter Type II superconductors above the lower critical field $H_{\mathrm{c} 1}$. The TGB phase predicted here for chiral polymers is similar to that already observed experimentally in chiral smectics [2]. The braided moiré state is qualitatively new, and its experimental observation would be of considerable interest. Surprising little is known about the intricate trajectories produced by iterated moiré maps shown in Figure 2. These could be studied experimentally via neutron diffraction in hexagonal columnar crystals with a dilute concentration of deuterated polymer strands. Numerical studies of the local fractal dimension and Lyapunov exponent are currently in progress.

It is a pleasure to acknowledge stimulating conversations with T. Lubensky, R. Meyer, P. Taylor, E. Thomas, and J. Toner. RDK was supported by National Science Foundation Grant No. PHY92-45317. DRN acknowledges the hospitality of Brandeis University, AT\&T Bell Laboratories, and Exxon Research and Engineering, as well as support from the Guggenheim Foundation and the National Science Foundation, through the Harvard Materials Research Laboratory and Grant No. DMR-91-15491 . 


\section{References}

[1] F. Livolant and Y. Bouligand, J. Phys. (Paris) 471813 (1986); For tilt and moiré boundaries in the non-biological polymer PBZO (poly-paraphenylene benzobisoxazole) see D.C. Martin and E.L. Thomas, Phil. Mag. A 64, 903 (1991).

[2] S.R. Renn and T.C. Lubensky, Phys. Rev. A 38, 2132 (1988); G. Srajer, R. Pindak, M.A. Waugh and J.W. Goodby, Phys. Rev. Lett. 64, 1545 (1990); see also P.G. de Gennes, Solid State Commun. 10, 753 (1972).

[3] P.G. de Gennes and J. Prost, The Physics of Liquid Crystals, Second ed., (Oxford University Press, New York, 1993).

[4] D.R. Nelson and J. Toner, Phys. Rev. B 24, 363 (1981) and references therein.

[5] J. Toner, Phys. Rev. A 271157 (1983).

[6] M.C. Marchetti and D.R. Nelson, Phys. Rev. B 411910 (1990).

[7] R.B. Meyer, Polymer Liquid Crystals, edited by A. Ciferri, W.R. Kringbaum and R.B. Meyer (Academic, New York, 1982) Chapter 6.

[8] E.M. Terentjev, Europhys. Lett. 23, 27 (1993).

[9] V.L. Indenbom and A.N. Orlov, Usp. Fiz. Nauk 76557 (1962) [ Sov. Phys. Uspekhi $5272(1962)]$.

[10] R.D. Kamien and D.R. Nelson, unpublished.

[11] M. Kléman and P. Oswald, J. Phys. (Paris) 43655 (1982).

[12] W.A. Bollman, Crystal Defects and Crystalline Interfaces, (Springer-Verlag, Berlin, 1970); J.P. Hirth and J. Lothe, Theory of Dislocations, Second ed. (Wiley, New York, 1982).

[13] D.C. Wright and N.D. Mermin, Rev. Mod. Phys. 61, 385 (1989). 


\section{Figure Captions}

Fig. 1. Phase diagram of a chiral polymer crystal. Insets are representative tilt (TGB) and moiré grain boundaries. Shaded lines are screw dislocations.

Fig. 2. A projected top view of the moiré map with rotation angle $\phi_{1} \approx 21.8^{\circ}$ iterated three times. The inset shows 20 random polymers paths resulting from the same map iterated 99 times.

Fig. 3. The moiré state. The thick tubes running in the $\hat{z}$ direction are polymers, while the dark lines are stacked honeycomb arrays of screw dislocations. 


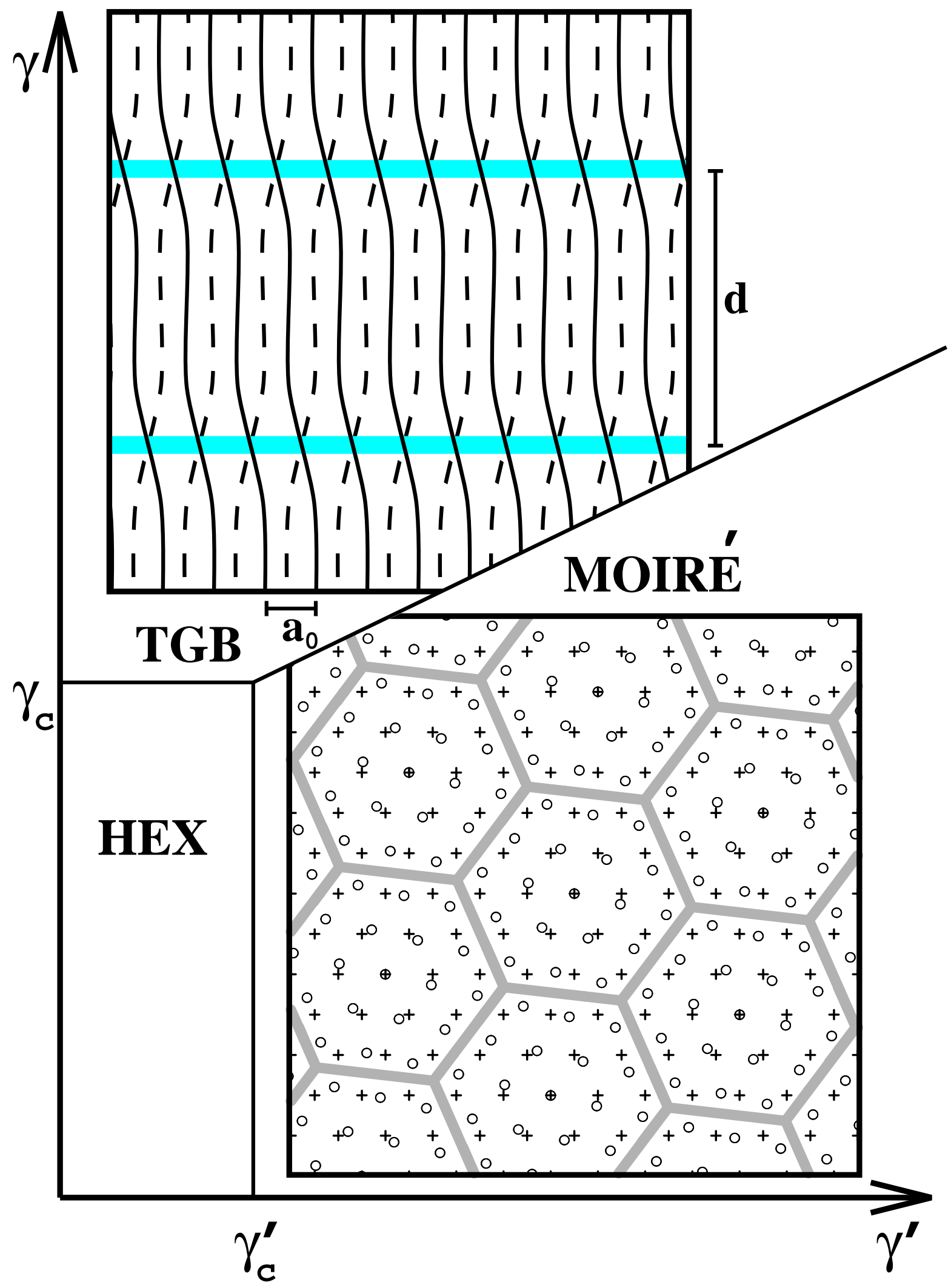

Figure 1 


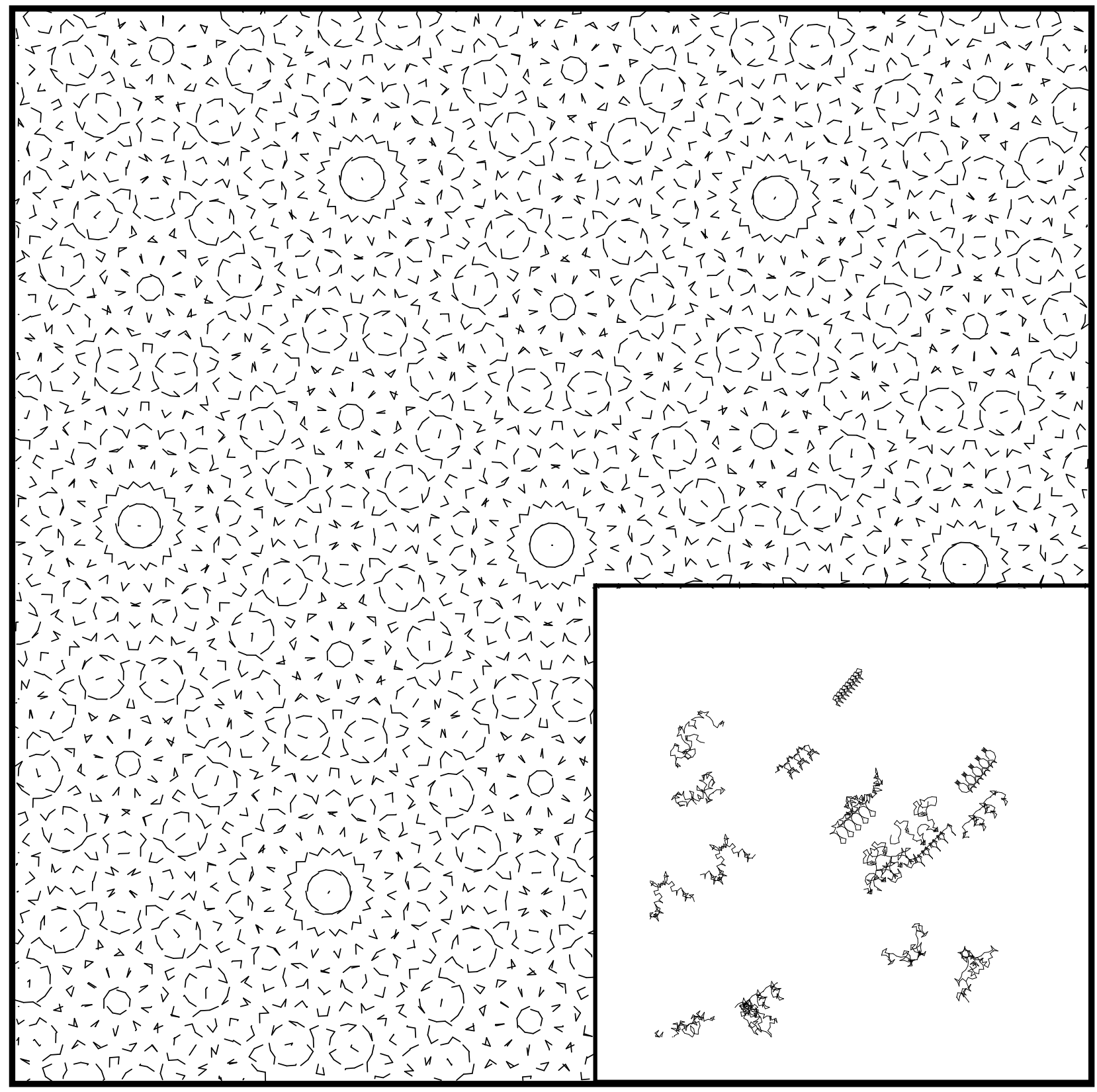

Figure 2 


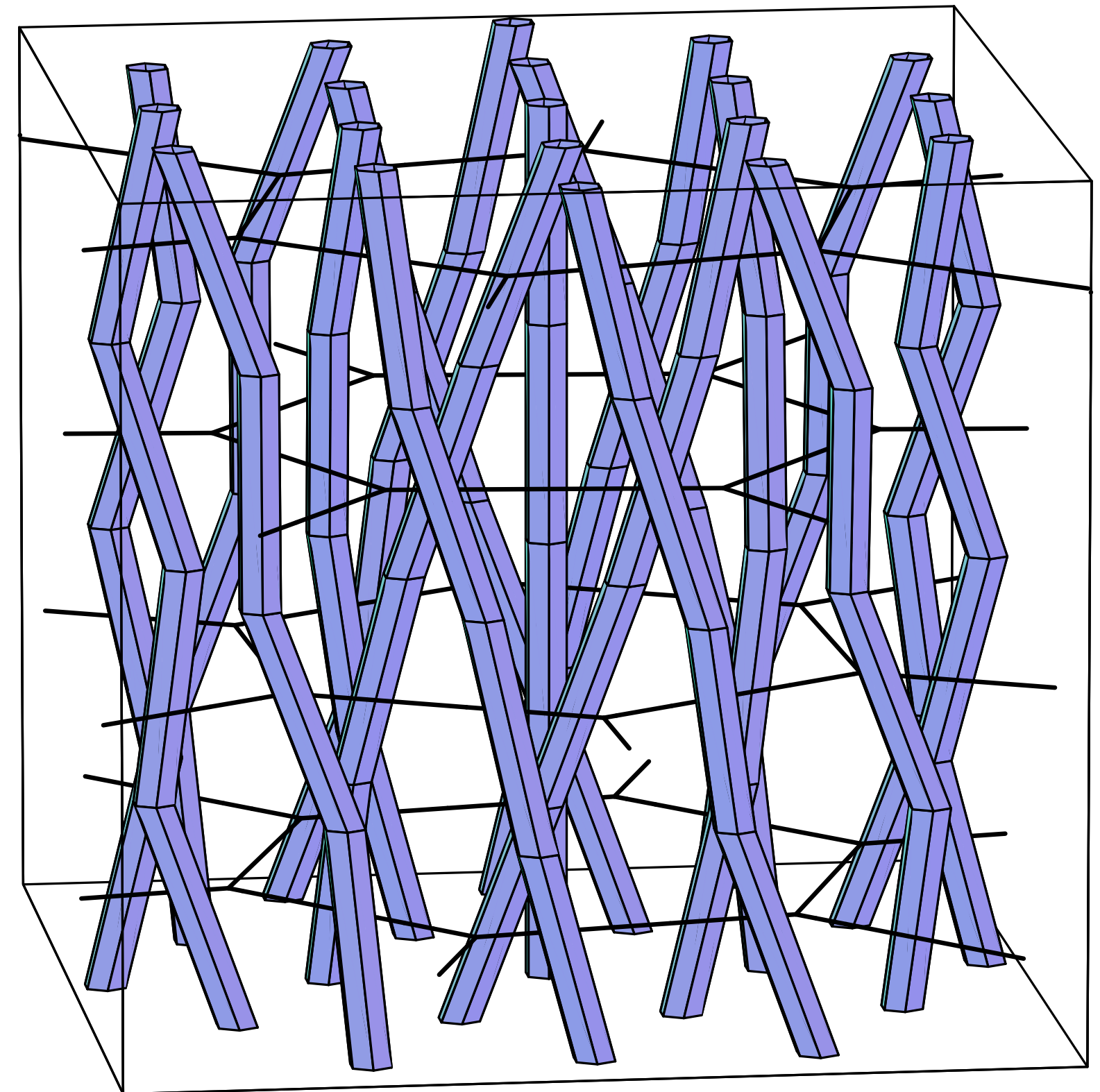

Figure 3 